\title{
Primary aldosteronism
}

\section{A Japanese perspective}

\author{
Fumitoshi Satoh • Ryo Morimoto • \\ Yoshitsugu Iwakura $\cdot$ Yoshikiyo Ono • \\ Masataka Kudo $\cdot$ Kei Takase $\cdot$ Sadayoshi Ito
}

Published online: 9 February 2011

(C) The Author(s) 2011. This article is published with open access at Springerlink.com

\begin{abstract}
Primary aldosteronism (PA) is the most common cause of secondary hypertension, accounting for $10 \%$ of all hypertension. Far from being benign, hypertension due to PA is associated with high cardiovascular morbidity and mortality. However, PA is still underdiagnosed in general practice. Recent reports strongly recommend that identifying patients with PA is cost-beneficial based on improved cardiovascular outcomes afforded by specific surgical and medical treatment. This review provides an update of PA including controversial aspects of diagnosis and treatment.
\end{abstract}

Keywords Aldosterone producing adenoma - Adrenal venous sampling $\cdot$ Laparoscopic adrenalectomy . Mineralocorticoid receptor antagonists · Primary aldosreronism

\section{What is primary aldosteronism?}

Primary aldosteronism (PA), also called primary hyperaldosteronism or Conn's syndrome, is characterized by overproduction of aldosterone mainly due to either a unilateral adrenocortical adenoma (APA) or to idiopathic bilateral adrenal hyperplasia (IHA). This excessive aldosterone secretion, which is relatively autonomous from the renin-angiotensin system and refractory to sodium loading, suppresses plasma renin and typically causes hypertension,

F. Satoh $(\bowtie) \cdot$ R. Morimoto $•$ Y. Iwakura $•$ Y. Ono $•$ M. Kudo $・$

S. Ito

Division of Nephrology, Endocrinology, and Vascular Medicine,

Tohoku University Hospital,

Sendai, Miyagi, Japan

e-mail: fsatoh@mail.tains.tohoku.ac.jp

K. Takase

Division of Radiology, Tohoku University Hospital,

Sendai, Miyagi, Japan sodium retention, and potassium excretion, which can lead to hypokalemia, hypomagnesemia and metabolic alkalosis.

\section{How common is PA?}

There is uncertainty about how common PA actually is. It was previously believed that the prevalence of PA was less than $1 \%$ of all hypertensive patients and that hypokalemia was necessary for detection of PA $[1,2]$. Some clinicians who still believe that the prevalence of PA is very low think that there may be no cost-benefit in routinely look for PA, and that testing for PA should be done only when drug therapy fails or young adults develop hypertension. However, using the plasma aldosterone concentration (PAC) to plasma renin activity (PRA) ratio (ARR) as the most reliable available screening test, many prospective and cross-sectional studies have shown that markedly increased detection rates for PA could be up to $10 \%$ of hypertensive patients, with most PA patients being normokalemic [3-8]. It is now widely recognized that primary aldosteronism (PA) is much more common than previously thought and that aldosterone excess has blood pressure (BP) - independent adverse cardiovascular and renal effects. These two major conceptual changes have prompted a lot of studies which have provided evidence for the role of PA in cardiovascular, renal and metabolic morbidity, and suggest that PA should be systematically diagnosed and treated.

\section{In which patient groups should detection of PA be recommended?}

The Endocrine Society USA issued clinical guidelines for the detection, diagnosis, and management of PA, which is a common, treatable and potentially curable disease [8]. The guidelines suggest measuring ARR to detect PA in patient 
groups with a high prevalence of PA, such as patients with severe or resistant hypertension, hypokalemia, hypertension with adrenal incidentaloma, early-onset hypertension and cerebrovascular accident at young age. This recommendation for selective detection acknowledges the costs associated with ARR testing of all hypertensive patients. However, the guidelines also suggest that the risk of missing or delaying the diagnosis of PA should be considerd in other hypertensive patients, as the consequences of this may be the later development of more severe and resistant hypertension resulting from failure to lower the level of aldosterone or block its actions. Several investigators have reported that duration of hypertension is a predictor of refractory hypertension after adrenalectomy for APA [9-11], suggesting that delayed diagnosis of PA may result in reduced effects of specific treatment for PA. Saving medical costs to detect PA might result in paying much higher costs due to cardiovascular complications.

The Japan Endocrine Society show a diagnostic and therapeutic guide on its homepage, which recommends that PA should be suspected in all patients with hypertension, especially untreated patients who are free from antihypertensive drugs that affect the ARR, a recommendation which recognizes the lower medical costs in Japan. $\beta$-blockers and clonidine can raise ARR by decreasing PRA more markedly than PAC; otherwise, ACE inhibitors (ACE-I), angiotensin II receptor blockers (ARBs), diuretics including spironolactone and dihydropyridine calcium channel blockers (CCBs) tend to reduce ARR [12]. Screening for PA among the newly diagnosed hypertensive patients is thus much easier. The prevalence of PA was reported to be $5.4 \%$ in 1,020 newly diagnosed Japanese hypertensive patients [5], and the prevalence of APA alone was also reported to be $4.8 \%$ in 1,125 newly diagnosed Italian hypertensive patients [7]. If a patient has resistant hypertension and suppressed PRA in spite of treatment with ACE-Is (or ARBs), CCBs and thiazides (or thiazide-like diuretics), the only physiological interpretation can be sodium retention due to PA. Nevertheless, in severe or resistant hypertension, withdrawal or replacement of antihypertensive drugs could be potentially harmful. Thus, we would like to stress that detection of PA should be done in mild or moderate stages of hypertension, preferably in newly diagnosed hypertensive patients.

\section{Is detection of PA beneficial for the patients?}

The complexity of detection and diagnosis of PA has raised questions concerning whether identifying PA patients is worth all the procedures, and whether specific treatment for PA reverses clinically meaningful excess in morbidity because of cardiovascular complications any better than usual antihypertensive therapy. Recent publications have answered these questions and have strongly pointed towards the affirmative. Patients with PA are at a higher risk for cardiovascular morbidity and mortality than patients with essential hypertension $(\mathrm{EH})$ matched for age, gender, and systolic and diastolic BP. Stroke, angina, myocardial infarction, chronic cardiac insufficiency and atrial fibrillation are obviously more common in patients with PA [13-16]. Furthermore, the excess in cardiovascular morbidity of PA patients was reversed in the long term after surgery and specific medical treatment [13].

Excessive aldosterone causes renal damage in patients with PA, leading to higher urinary albumin excretion (UAE) than in matched patients with EH [17, 18]. This higher UAE in PA patients is associated with a state of glomerular hyperfiltration resulting from aldosterone-induced sodium retention and volume expansion, and is reversed by unilateral adrenalectomy in APA or by spironolactone treatment [18].

\section{Is there any association between PA and metabolic syndrome?}

There is ongoing debate concerning whether there is an association between PA and metabolic syndrome that would explain the excess cardiovascular morbidity. The affirmative reports suggest that metabolic syndrome is more prevalent in patients with PA than in others [19] and that metabolic abnormality in patients with APA improves after surgery [20]. In contrast to these findings, a very recent retrospective study found no difference in fasting glucose levels, lipid profile or prevalence of diabetes between 460 patients with PA and 1363 controls with EH [21].

\section{Confirmatory tests for PA}

The Endocrine Society guidelines recommend that either the oral salt-loading test (OSLT), saline infusion testing (SIT), fludrocortisone suppression test or captopril challenge test (CCT) should be performed to confirm PA in patients with an elevated ARR under conditions of minimum pharmacological effects on the renin-angiotensin-aldosterone system [8]. These four tests may differ in terms of sensitivity, specificity and reliability. Therefore, the choice of a confirmatory test is determined by cost, patient compliance and local expertise, and still remains a matter of debate $[19,20]$. The diagnostic and therapeutic guide for PA from the Japan Endocrine Society recommends three confirmatory tests: furosemideupright test (FUT), CCT and SIT. Of them, FUT used to be performed in Japan, but it has a slightly lower sensitivity and specificity and may involve considerable physical stress, and now widely replaced by CCT, which can be performed in the outpatient clinic thanks to its simplicity [22] although there is a controversial report [23]. 


\section{Subtype differentiation of PA}

The key diagnostic step in patients who want to pursue a surgical cure for PA is to distinguish between unilateral and bilateral aldosterone hypersecretion. Many reports suggest that despite recent advances in imaging technology, small APA may not be detectable on adrenaldirected computed tomography (CT) or magnetic resonance imaging (MRI), and that adrenal non-functioning incidentalomas may coexist with IHA [24-27]. A very recent meta-analysis of subtype differentiation of PA by adrenal venous sampling (AVS), and CT or MRI, concluded that imaging did not detect laterality of hyperaldosteronism in $37.8 \%$ of the cases based on the data from 950 PA patients in 38 reports when AVS was used as the standard test for localization and diagnosis [28]; clinical decisions based solely on imaging methods can thus lead to inappropriate treatment. Although AVS is the gold standard for localization of hyperaldosteronism, the lack of standardized protocols and the other low rate of successful cannulation continue to be challenging issues.

\section{New strategies involving specific medical treatment for PA}

Eplerenone, a selective mineralocorticoid antagonist, has been reported to be as effective as spironolactone in reducing BP in patients with PA without sex steroidrelated side effects [29]. Eplerenone, at approximately 50 cents (US) for a $25 \mathrm{mg}$ tablet in Japan, is now widely used to treat patients with PA in Japan with some restrictions. Hopefully, the usage of eplerenone for PA may soon be considered in other countries where usage is less common. Specific aldosterone synthase inhibitors currently under phase II clinical trials may become a new treatment modality for PA without inhibiting production of cortisol or sex steroids [30].

\section{Perspectives}

Given the large number of patients with PA, comprehensive screening programs and referral to specific centers need to be developed by an interested group of endocrinologists, hypertension specialists, internists, radiologists and surgeons within each local community.

Open Access This article is distributed under the terms of the Creative Commons Attribution Noncommercial License which permits any noncommercial use, distribution, and reproduction in any medium, provided the original author(s) and source are credited.

\section{References}

1. Ganguly A. Primary aldosteronism. N Engl J Med. 1998;339:182834.

2. Kaplan NM. Hypokalemia in the hypertensive patient, with observations on the incidence of primary aldosteronism. Ann Intern Med. 1967;66:1079-90.

3. Fardella CE, Mosso L, Gómez-Sánchez C, et al. Primary hyperaldosteronism in essential hypertensives: prevalence, biochemical profile, and molecular biology. J Clin Endocrinol Metab. 2000;85:1863-7.

4. Williams JS, Williams GH, Raji A, Jeunemaitre X, et al. Prevalence of primary hyperaldosteronism in mild to moderate hypertension without hypokalaemia. J Hum Hypertens. 2006;20:129-36.

5. Nishikawa T, Omura M. Clinical characteristics of primary aldosteronism: its prevalence and comparative studies on various causes of primary aldosteronism in Yokohama Rosai Hospital. Biomed Pharmacother. 2000;54 Suppl 1:83-5.

6. Stowasser M, Gordon RD. Primary aldosteronism-careful investigation is essential and rewarding. Mol Cell Endocrinol. 2004;217:33-9.

7. Rossi GP, Bernini G, Caliumi C, et al. A prospective study of the prevalence of primary aldosteronism in 1, 125 hypertensive patients. J Am Coll Cardiol. 2006;48:2293-300.

8. Funder JW, Carey RM, Fardella C, et al. Case detection, diagnosis, and treatment of patients with primary aldosteronism: an endocrine society clinical practice guideline. J Clin Endocrinol Metab. 2008;93:3266-81.

9. Streeten DH, Anderson Jr GH, Wagner S. Effect of age on response of secondary hypertension to specific treatment. Am J Hypertens. 1990;3(5 Pt 1):360-5.

10. Celen O, O’Brien MJ, Melby JC, Beazley RM. Factors influencing outcome of surgery for primary aldosteronism. Arch Surg. 1996;131:646-50.

11. Rossi GP, Bolognesi M, Rizzoni D, et al. Vascular remodeling and duration of hypertension predict outcome of adrenalectomy in primary aldosteronism patients. Hypertension. 2008;51:1366-71.

12. Mulatero P, Rabbia F, Milan A, et al. Drug effects on aldosterone/ plasma renin activity ratio in primary aldosteronism. Hypertension. 2002;40:897-902.

13. Milliez P, Girerd X, Plouin PF, Blacher J, Safar ME, Mourad JJ. Evidence for an increased rate of cardiovascular events in patients with primary aldosteronism. J Am Coll Cardiol. 2005;45:12438.

14. Catena C, Colussi G, Nadalini E, et al. Cardiovascular outcomes in patients with primary aldosteronism after treatment. Arch Intern Med. 2008;168:80-5.

15. Born-Frontsberg E, Reincke M, Rump LC, et al. Cardiovascular- and cerebrovascular comorbidites of hypokalemic- and normokalemic primary aldosteronism: results of the German Conn's Registry. 2009. J Clin Endocrinol Metab. 2009;94:1125-30.

16. Stowasser M, Sharman J, Leano R, et al. Evidence for abnormal left ventricular structure and function in normotensive individuals with familial hyperaldosteronism type I. J Clin Endocrinol Metab. 2005;90:5070-6.

17. Rossi GP, Bernini G, Desideri G, et al. Renal damage in primary aldosteronism: results of the PAPY Study. Hypertension. 2006;48:232-8.

18. Sechi LA, Di Fabio A, Bazzocchi M, Uzzau A, Catena C. Intrarenal hemodynamics in primary aldosteronism before and after treatment. J Clin Endocrinol Metab. 2009;94:1191-7.

19. Fallo F, Veglio F, Bertello C, et al. Prevalence and characteristics of the metabolic syndrome in primary aldosteronism. J Clin Endocrinol Metab. 2006;91:454-9. 
20. Giacchetti G, Ronconi V, Turchi F, et al. Aldosterone as a key mediator of the cardiometabolic syndrome in primary aldosteronism: an observational study. J Hypertens. 2007;25:177-86.

21. Matrozova J, Steichen O, Amar L, Zacharieva S, Jeunemaitre X, Plouin PF. Fasting plasma glucose and serum lipids in patients with primary aldosteronism: a controlled cross-sectional study. Hypertension. 2009;53:605-10.

22. Rossi GP, Belfiore A, Bernini G, et al. Comparison of the captopril and the saline infusion test for excluding aldosteroneproducing adenoma. Hypertension. 2007;50:424-31.

23. Mulatero P, Bertello C, Garrone C, et al. Captopril test can give misleading results in patients with suspect primary aldosteronism. Hypertension. 2007;50:e26-7.

24. Young WF, Stanson AW, Thompson GB, Grant CS, Farley DR, van Heerden JA. Role for adrenal venous sampling in primary aldosteronism. Surgery. 2004;136:1227-35.

25. Doppman JL, Gill Jr JR, Miller DL, et al. Distinction between hyperaldosteronism due to bilateral hyperplasia and unilateral aldosteronoma: reliability of CT. Radiology. 1992;184:677-82.
26. Omura M, Sasano H, Saito J, Yamaguchi K, Kakuta Y, Nishikawa T. Clinical characteristics of aldosterone-producing microadenoma, macroadenoma, and idiopathic hyperaldosteronism in 93 patients with primary aldosteronism. Hypertens Res. 2006;29:883-9.

27. Satoh F, Abe T, Tanemoto $M$, et al. Localization of aldosterone-producing adrenocortical adenomas: significance of adrenal venous sampling. Hypertens Res. 2007;30:108395.

28. Kempers MJ, Lenders JW, van Outheusden L, et al. Systematic review: diagnostic procedures to differentiate unilateral from bilateral adrenal abnormality in primary aldosteronism. Ann Intern Med. 2009;151:357-8.

29. Karagiannis A, Tziomalos K, Papageorgiou A, et al. Spironolactone versus eplerenone for the treatment of idiopathic hyperaldosteronism. Expert Opin Pharmacother. 2008;9:509-15.

30. Jansen PM, van den Meiracker AH, Jan Danser AH. Aldosterone synthase inhibitors: pharmacological and clinical aspects. Curr Opin Investig Drugs. 2009;10:319-26. 